\title{
Population Pharmacokinetic Modeling of the Enterohepatic Recirculation of Fimasartan in Rats, Dogs, and Humans
}

\author{
Tae Hwan Kim, ${ }^{1}$ Soyoung Shin, ${ }^{2}$ Cornelia B. Landersdorfer, ${ }^{3,4}$ Yong Ha Chi, ${ }^{5}$ Soo Heui Paik, ${ }^{5}$ Jayhyuk Myung, ${ }^{5}$ \\ Rajbharan Yadav, ${ }^{3}$ Stefan Horkovics-Kovats, ${ }^{6}$ Jürgen B. Bulitta, ${ }^{3,4,8}$ and Beom Soo Shin ${ }^{7,8}$
}

Received 11 December 2014; accepted 30 March 2015; published online 20 May 2015

\begin{abstract}
Enterohepatic recirculation (EHC) can greatly enhance plasma drug exposures and therapeutic effects. This study aimed to develop a population pharmacokinetic model that can simultaneously characterize the extent and time-course of EHC in three species using fimasartan, a novel angiotensin II receptor blocker, as a model drug. All fimasartan plasma concentration profiles in 32 rats (intravenous doses, $0.3-3 \mathrm{mg} / \mathrm{kg}$; oral doses, $1-10 \mathrm{mg} / \mathrm{kg}$ ), $34 \mathrm{dogs}$ (intravenous doses, $0.3-1 \mathrm{mg} / \mathrm{kg}$; oral doses, $1-10 \mathrm{mg} / \mathrm{kg}$ ), and 42 healthy volunteers (single or multiple oral doses, $20-480 \mathrm{mg}$ ) were determined via liquid chromatography-tandem mass spectrometry (LCMS/MS) and simultaneously modeled in S-ADAPT. The proposed model quantitatively characterized EHC in three species after oral and intravenous dosing. The median (range) fraction of drug undergoing recirculation was $76.3 \%(64.9-88.7 \%)$ in rats, $33.3 \%(24.0-45.9 \%)$ in dogs, and $65.6 \%(56.5-72.0 \%)$ in humans. In the presence compared with the absence of EHC, the area under the curve in plasma was predicted to be 4.22 -fold (2.85-8.85) as high in rats, 1.50-fold (1.32-1.85) in dogs, and 2.91-fold (2.30-3.57) in humans. The modeled oral bioavailability in rats (median (range), 38.7\% (20.0-59.8\%)) and dogs (median, $7.13 \%$ to $15.4 \%$, depending on the formulation) matched the non-compartmental estimates well. In humans, the predicted oral bioavailability was $25.1 \%(15.1-43.9 \%)$ under fasting and $18.2 \%(12.2-31.0 \%)$ under fed conditions. The allometrically scaled area under the curve predicted from rats was $420 \mathrm{ng} \cdot \mathrm{h} / \mathrm{mL}$ for $60 \mathrm{mg}$ fimasartan compared with $424 \pm 63 \mathrm{ng} \cdot \mathrm{h} / \mathrm{mL}$ observed in humans. The developed population pharmacokinetic model can be utilized to characterize the impact of EHC on plasma drug exposure in animals and humans.
\end{abstract}

KEY WORDS: animal to human scaling; enterohepatic recirculation; fimasartan; population pharmacokinetics; S-ADAPT.

\section{INTRODUCTION}

Enterohepatic recirculation (EHC) involves drug in the liver that is excreted via bile into the small intestine and subsequently reabsorbed. It is known to be a common

Jürgen B. Bulitta and Beom Soo Shin contributed equally to this work. Electronic supplementary material The online version of this article (doi:10.1208/s12248-015-9764-2) contains supplementary material, which is available to authorized users.

${ }^{1}$ School of Pharmacy, Sungkyunkwan University, Suwon, Gyeonggi-do, Korea.

${ }^{2}$ Department of Pharmacy, College of Pharmacy, Wonkwang University, Iksan, Jeonbuk, Korea.

${ }^{3}$ Drug Delivery, Disposition and Dynamics, Monash Institute of Pharmaceutical Sciences, Monash University (Parkville campus), Parkville, VIC 3052, Australia.

${ }^{4}$ School of Pharmacy and Pharmaceutical Sciences, State University of New York at Buffalo, Buffalo, New York, USA.

${ }^{5}$ Central Research Institute, Boryung Pharm. Co., Ltd., Seoul, Korea.

${ }^{6}$ Sandoz GmbH, Kundl, Austria.

${ }^{7}$ College of Pharmacy, Catholic University of Daegu, Gyeongsan-si, Gyeongbuk, Korea.

${ }^{8}$ To whom correspondence should be addressed. (e-mail: bsshin@cu.ac.kr; j@bulitta.com) pharmacokinetic (PK) characteristic of angiotensin II receptor blockers (ARBs) such as fimasartan, losartan, irbesartan, and telmisartan (1-5) and other drug classes (6-8) with considerable biliary excretion. Extensive EHC leads to multiple peaks (or "shoulders") in the plasma concentration time profiles and a prolonged terminal halflife. While second peaks after oral administration can be caused by complex absorption kinetics (9-12), the presence of multiple peaks after intravenous (IV) dosing strongly suggests the presence of EHC. The increased drug exposure and prolonged terminal half-life due to EHC can be pharmacologically important and enhance therapeutic effects (8).

Fimasartan (Kanarb®, Boryung Pharm. Co., Ltd) is a novel ARB approved by the Korean Ministry of Food and Drug Safety (MFDS) in 2010 for the treatment of mild to moderate hypertension. Fimasartan provides a selective angiotensin II type 1 (AT1) receptor antagonist effect, and its affinity to the AT1 receptor is greater than that of losartan (13). In contrast to other ARBs, fimasartan did not show partial agonistic effects on the angiotensin II receptor in animal models (13). Consequently, phases II and III studies demonstrated a higher potency and stronger efficacy of fimasartan compared with losartan with a rapid onset of antihypertensive effect $(14,15)$. 
A recent preclinical study (16) indicated that fimasartan is mainly excreted via feces; and multiple peaks in the plasma concentration-time profiles were observed both after oral and IV administration, suggesting the presence of EHC. Multiple plasma concentration peaks were also present after oral administration of fimasartan in clinical trials (17-22), indicating that EHC impacts on human PK and potentially pharmacodynamics (PD). It is therefore important to quantitatively understand how EHC affects the systemic fimasartan exposure to enable animal to human scaling and ultimately the prediction of first-in-human (FIH) PK. However, predicting the PK of compounds undergoing considerable EHC is not straightforward (23).

Models for EHC have been developed for various drugs using plasma concentrations after a single IV or oral dose $(24,25)$. These models typically describe the time of onset of EHC by a single on/off switch (i.e., a "lag-time") (26) or characterize the periodic time-course of EHC via a sine function $(27,28)$. To our knowledge, all published models only characterized EHC in one species and did not fit plasma concentration time profiles in the presence of EHC after multiple dosing.

Our aims were to develop a population model that can quantitatively describe and compare EHC in rats, dogs, and humans and characterize the impact of EHC following IV and single and multiple oral doses. Additionally, we sought to compare the rate and extent of absorption for three different fimasartan formulations and predict absolute oral bioavailability in humans. This approach is expected to provide detailed insights into the extent and time-course of EHC and thereby support the optimization of dosage regimens for patients.

\section{MATERIALS AND METHODS}

\section{Chemicals and Reagents}

Fimasartan and the internal standard (BR-A-563) (29) were supplied by Boryung Pharm. Co., Ltd. (Seoul, Korea). Zoletil 50® (tiletamine/zolazepam $=125 / 125 \mathrm{mg}$ ) was purchased from Virbac Laboratory (Carros cedex, France), high-performance liquid chromatography (HPLC)-grade acetonitrile, methanol, and distilled water from Mallinckrodt Baker (Phillipsburg, NJ), and formic acid from Aldrich Chemicals (Milwaukee, WI). Heparin sodium and saline were obtained from Choong Wae Pharma (Seoul, Korea).

\section{Data Sets}

The PK profiles of fimasartan from 32 rats (intravenous doses, $0.3-3 \mathrm{mg} / \mathrm{kg}$; oral doses, 1-10 mg/kg), $34 \mathrm{dogs}$ (intravenous doses, $0.3-1 \mathrm{mg} / \mathrm{kg}$; oral doses, $1-10 \mathrm{mg} / \mathrm{kg}$ ), and 42 healthy volunteers (single or multiple oral doses, 20$480 \mathrm{mg}$ ) were assessed. The rat (16) and human (21) data have been published previously, and the dog data were generated in the present study and are presented for the first time. The data sets included in our modeling analysis are summarized in the supplement Table S1.

\section{Animal Experiments}

All animal studies were conducted following the Guidelines for the Care and Use of Animals. The studies were approved by the Ethics Committee for the Treatment of Laboratory Animals at Boryung Pharm. Co., Ltd. and the Catholic University of Daegu.

Rat Studies. Sprague-Dawley rats were anesthetized with $20 \mathrm{mg} / \mathrm{kg}$ Zoletil 50® (tiletamine $\mathrm{HCl} 125 \mathrm{mg} / 5 \mathrm{~mL}$ +zolazepam $\mathrm{HCl} 125 \mathrm{mg} / 5 \mathrm{~mL}$ ) and cannulated in the right jugular and femoral veins for IV injection and the right jugular vein for rats receiving oral dosing as described previously (16).

Dog Studies. Beagle dogs (24 males, 10 females, 5.5 months old) were obtained from Marshall BioResources (Beijing, China). For IV injection, a catheter was placed and fixed in the cephalic vein of the front leg. Fimasartan was dissolved in distilled water, and the drug solution ( 0.3 and $1 \mathrm{mg} / \mathrm{kg} ; n=6$ for each group) was dosed as a bolus via the catheter followed by flushing. Blood samples of $3 \mathrm{~mL}$ were collected from the cephalic vein at pre-dose (within $5 \mathrm{~min}$ ) and at 5, 10, 20, and $30 \mathrm{~min}$ and 1, 1.5, 2, 3, 4, 6, 8, 12, 16, 20, 24,48 , and $72 \mathrm{~h}$ after IV injection.

For oral administration, three different fimasartan formulations were used; i.e., an oral solution, capsule, and tablet. For the solution, fimasartan was dissolved in distilled water (dosed at $3 \mathrm{mg} / \mathrm{kg}, n=8)$. The capsules were filled with appropriate amounts of fimasartan using doses of 1,3 , and $10 \mathrm{mg} / \mathrm{kg}(n=3$, each). The tablet was identical to that available on the market (60 mg/tablet, equivalent to a dose of $6 \mathrm{mg} / \mathrm{kg}$ in dogs, $n=5$ ). All dogs were studied under fasting conditions. After dosing, $3 \mathrm{~mL}$ of blood were collected from the cephalic vein at pre-dose (within $5 \mathrm{~min}$ ), and at 10, 20, and $30 \mathrm{~min}$ and 1, 1.5, 2, 3, 4, 6, 8, 12, 16, 20, 24, 48, and $72 \mathrm{~h}$. Plasma was obtained by centrifugation at $15,000 \times g$ at $4^{\circ} \mathrm{C}$ for $10 \mathrm{~min}$ and immediately frozen and stored at $-70^{\circ} \mathrm{C}$ until analysis.

\section{Clinical Studies}

The single dose PK of fimasartan was assessed in a randomized, double-blind study (21) following oral administration of a tablet formulation containing 20,60, 120, 240, and $480 \mathrm{mg}$ fimasartan to 30 healthy subjects (six subjects per dose group). Five of the six subjects in the 240-mg group also received $240 \mathrm{mg}$ fimasartan in the fed state after a wash-out period of 7 days as described previously (21). We additionally used data from a randomized, double-blind, multiple-dose PK study which included 12 subjects receiving oral doses of 120 or $360 \mathrm{mg}$ fimasartan every $24 \mathrm{~h}$ for 7 days. Detailed PK profiles were obtained on days 1 and 7 (21).

\section{Determination of Fimasartan Concentration by LC-MS/MS}

The fimasartan plasma concentrations in rats (16) and humans (21) were determined by liquid chromatographytandem mass spectrometry (LC-MS/MS) as described previously. Fimasartan plasma concentrations in dogs were determined by a newly developed LC-MS/MS assay. Briefly, $75 \mu \mathrm{L}$ of $1 \%$ formic acid, $1 \mathrm{~mL}$ of ethyl acetate and hexane mixture 
(80:20, $v / v)$, and $25 \mu \mathrm{L}$ of internal standard solution (BR-A563 at $200 \mathrm{ng} / \mathrm{mL}$ in $50 \%$ methanol) were added to $250 \mu \mathrm{L}$ of the plasma samples. The tubes were vigorously shaken with a vortex mixer for $5 \mathrm{~min}$ followed by centrifugation for $5 \mathrm{~min}$ at $15,000 \times g$ at $4^{\circ} \mathrm{C}$. The supernatant $(\sim 900 \mu \mathrm{L})$ was transferred to a polypropylene tube and evaporated under $\mathrm{N}_{2}$ at $40^{\circ} \mathrm{C}$. The residue of each evaporated sample was reconstituted with $80 \mu \mathrm{L}$ of mobile phase and mixed for $5 \mathrm{~min}$. The reconstituted solution was then transferred to a $2 \mathrm{~mL}$ microfilter tube and centrifuged. The filtered solution $(10 \mu \mathrm{L})$ was injected into the LC-MS/MS system.

The LC-MS/MS instrument comprised an API 3000 mass spectrometer (Applied Biosystems/MDS Sciex, Toronto, Canada) coupled with an Agilent 1100 HPLC (Agilent Technologies, Santa Clara, CA, USA). Fimasartan was separated on a Capcell Pak $\mathrm{C}_{18}$ column $50 \times 2.0 \mathrm{~mm}$, i.d., $3 \mu \mathrm{m}$ (Shiseido, Tokyo, Japan). The isocratic mobile phase composition was a mixture of $0.1 \%$ acetic acid in acetonitrile and $0.1 \%$ acetic acid in $1 \mathrm{mM}$ ammonium acetate $(70: 30, v / v)$. The flow rate of the mobile phase was set to $0.2 \mathrm{~mL} / \mathrm{min}$, and the column oven temperature was $30^{\circ} \mathrm{C}$. The mass spectrometer was operated using electron spray ionization (ESI) with negative ion mode. The transition of the precursors to the product ion was monitored at $500.7 \rightarrow 220.95$ for fimasartan and $524.16 \rightarrow 205.05$ for the internal standard (BR-A-563).

The lower limit of quantification of the LC-MS/MS assay was $0.2 \mathrm{ng} / \mathrm{mL}$ for rat and $\operatorname{dog}$ plasma and $0.4 \mathrm{ng} / \mathrm{mL}$ for human plasma. The method was validated by using the matrix-matched quality control (QC) samples (including QC samples at the lower limit of quantification). The intra- and inter-day accuracy and precision ranged from $90.8 \%$ to $108.0 \%$ and $2.4 \%$ to $13.4 \%$ for rat plasma, from $93.9 \%$ to $105.9 \%$ and $1.6 \%$ to $7.2 \%$ for dog plasma, and from $94.8 \%$ to $105.5 \%$ and $3.2 \%$ to $11.9 \%$ for human plasma.

\section{Population Pharmacokinetic Modeling}

The overall model development process is summarized in Fig. 1. Initially, we developed models for rats, dogs and humans separately (step 1). Then the final model was developed to simultaneously describe the data for all species, doses, and routes of administration (step 2). To illustrate the capabilities of the proposed EHC model, we additionally examined whether the human PK profile could be predicted via allometric scaling using the models developed based on the rat and dog data (step 3).

Structural Model. Drug dissolution (rate constant: $k_{\text {dis }}$; for the tablet and capsule formulations), transfer from the stomach into the gut compartment $\left(k_{\text {lag }}\right)$, and absorption from the gut compartment $\left(k_{\text {abs }}\right)$ were described by first-order processes (Fig. 2). The differential equations for undissolved drug, i.e., for the tablet and capsule formulations $\left(X_{\text {Solid }}\right)$ and dissolved drug in the stomach $\left(X_{\text {Stom }}\right)$ were:

$\frac{\mathrm{d} X_{\text {Solid }}}{\mathrm{d} t}=-k_{\mathrm{dis}} \cdot X_{\text {Solid }}$

$\frac{\mathrm{d} X_{\text {Stom }}}{\mathrm{d} t}=F_{\text {Cap }} \cdot F_{\text {Food, Rel }} \cdot k_{\text {dis }} \cdot X_{\text {Solid }}-k_{\text {lag }} \cdot X_{\text {Stom }}$
The $F_{\text {Cap }}$ is the relative bioavailability of the capsule formulation in dogs compared with the oral tablet and oral solution ( $F_{\text {Cap }}$ was fixed to 1.0 for the tablet and solution). The $F_{\text {Food, Rel }}$ represents the relative bioavailability of the tablet (240 $\mathrm{mg}$ dose) in the fed compared with the fasting state in humans ( $F_{\text {Food, Rel }}$ was fixed to 1.0 for all doses in the fasting state). All initial conditions were zero, unless stated otherwise (Fig. 2 shows compartments receiving bolus doses). Our model was simplified, as fimasartan was absorbed from the gut either into the central or the liver compartment and the associated fraction entering the liver compartment $\left(\mathrm{Fr}_{\text {Liver }}\right)$ was estimated. The differential equation for fimasartan in gut $\left(X_{\mathrm{Gut}}\right)$ was:

$\frac{\mathrm{d} X_{\mathrm{Gut}}}{\mathrm{d} t}=k_{\mathrm{lag}} \cdot X_{\mathrm{Stom}}-k_{\mathrm{abs}} \cdot X_{\mathrm{Gut}}+k_{\mathrm{Bile}-\mathrm{Gut}}(t) \cdot X_{\text {Bile }}$

The $k_{\text {Bile-Gut }}(t)$ is the time-dependent first-order rate constant (see below) for the transfer of fimasartan from bile into gut.

The systemic disposition of fimasartan was described by linear models with a central and one or two peripheral compartment(s). There was no clearance directly from the central compartment; instead, fimasartan transferred from the central into the liver compartment (clearance: $\mathrm{CLd}_{1-\mathrm{Liv}}$ ). The differential equations for the amounts of drug in the central $\left(X_{1}\right)$, shallow peripheral $\left(X_{2}\right)$, and deep peripheral compartment $\left(X_{3}\right)$ were:

$$
\begin{aligned}
& \begin{aligned}
\frac{\mathrm{d} X_{1}}{\mathrm{~d} t}= & \left(1-\mathrm{Fr}_{\text {Liver }}\right) \cdot k_{\mathrm{abs}} \cdot X_{\mathrm{Gut}}-\left(\mathrm{CLd}_{\text {Shallow }}+\mathrm{CLd}_{\text {Deep }}\right) \cdot C_{1} \\
& +\mathrm{CLd}_{\text {Shallow }} \cdot C_{2}+\mathrm{CLd}_{\text {Deep }} \cdot C_{3}-\mathrm{CLd}_{1-\mathrm{Liv}} \cdot C_{1}
\end{aligned} \\
& \frac{\mathrm{d} X_{2}}{\mathrm{~d} t}=\operatorname{CLd}_{\text {Shallow }} \cdot\left(C_{1}-C_{2}\right)
\end{aligned}
$$

$\frac{\mathrm{d} X_{3}}{\mathrm{~d} t}=\operatorname{CLd}_{\text {Deep }} \cdot\left(C_{1}-C_{3}\right)$

The $C_{1}, C_{2}$, and $C_{3}$ are the fimasartan concentrations in the respective compartment and $\mathrm{CLd}_{\text {Shallow }}$ and $\mathrm{CLd}_{\text {Deep }}$ are the distribution clearances to the shallow and deep peripheral compartment. Fimasartan in the liver compartment was either eliminated (first-order rate constant: $k_{\mathrm{el}}$ ) or transferred into the bile compartment $\left(k_{\text {Liv-Bile }}\right)$ :

$$
\begin{aligned}
\frac{\mathrm{d} X_{\text {Liver }}}{\mathrm{dt}}= & \operatorname{Fr}_{\text {Liver }} \cdot \mathrm{k}_{\mathrm{abs}} \cdot \mathrm{X}_{\mathrm{Gut}} \\
& +\mathrm{CLd}_{1-\text { Liv }} \cdot \mathrm{C}_{1}-\left(\mathrm{k}_{\mathrm{el}}+\mathrm{k}_{\text {Liv-Bile }}\right) \cdot \mathrm{X}_{\text {Liver }}
\end{aligned}
$$

Transfer of fimasartan from bile into the gut compartment, which represents regular gall bladder emptying, was described by an inducible first-order process $\left(k_{\text {Bile-Gut }}\right.$ $(t))$ :

$\frac{\mathrm{d} X_{\text {Bile }}}{\mathrm{d} t}=k_{\text {Liv-Bile }} \cdot X_{\text {Liver }}-k_{\text {Bile-Gut }}(t) \cdot X_{\text {Bile }}$ 


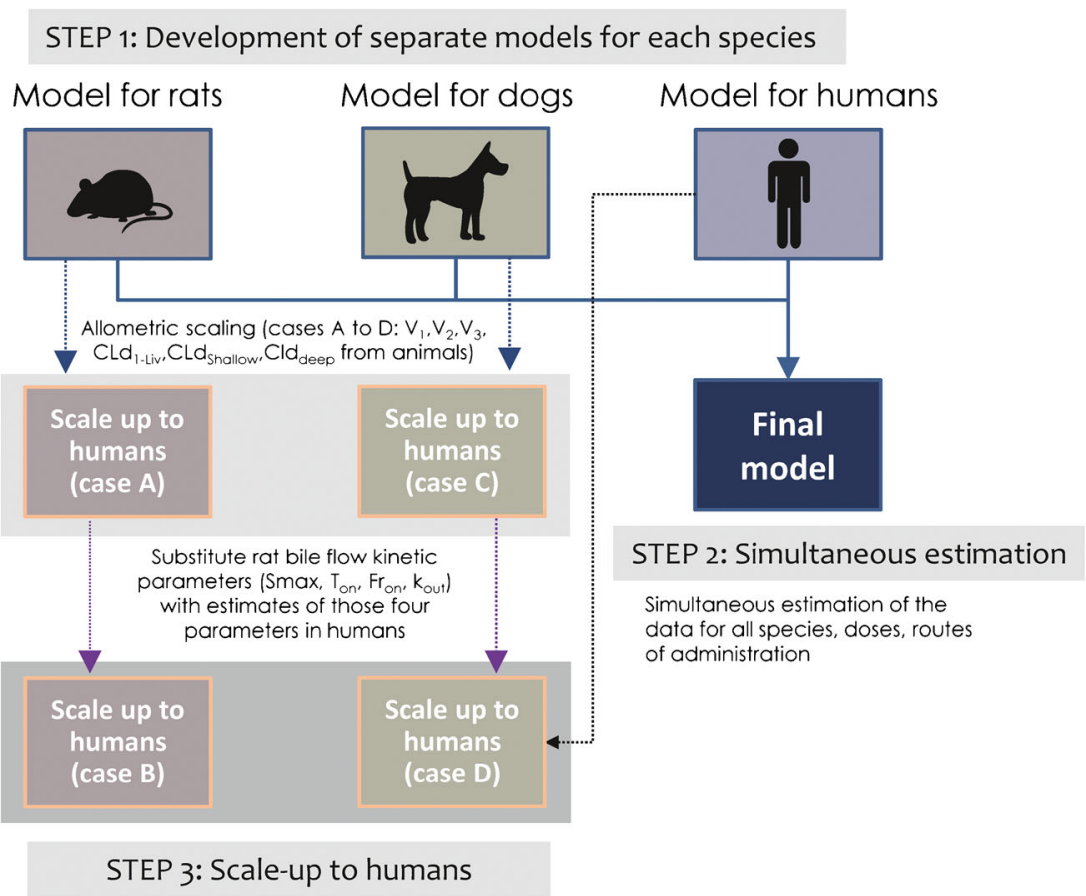

Fig. 1. Overall model development strategy. Initially, population PK models for rats, dogs, and humans were developed separately based on the same structural model for each species (step 1). Then, the final model was developed to simultaneously describe the data for all species, doses, and routes of administration (step 2). Additionally, we examined whether human PK profiles could be predicted by allometric scaling based on the rat model and the dog model (step 3)

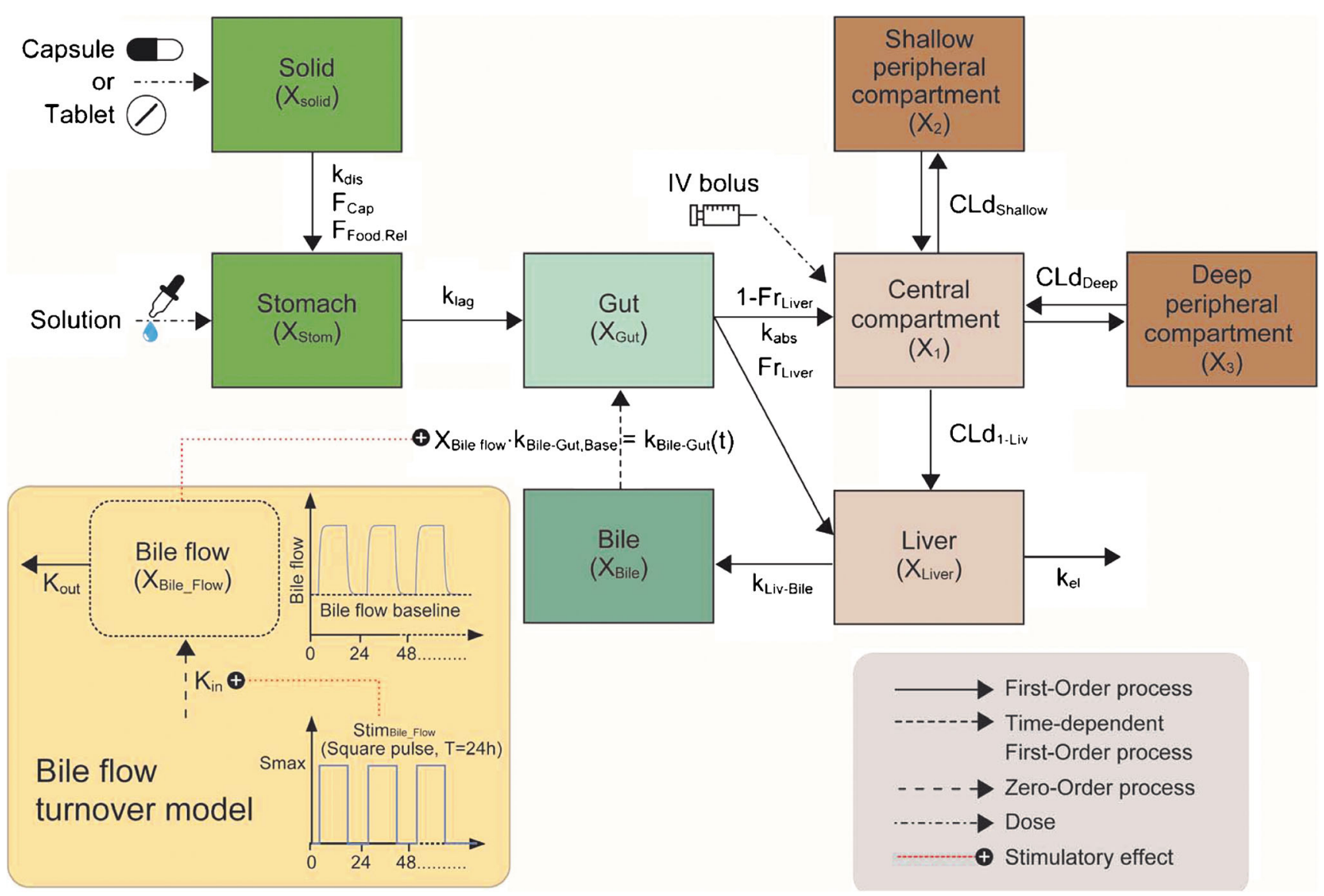

Fig. 2. Structural model for the absorption, disposition and enterohepatic recirculation of fimasartan in rats, dogs, and humans 
The first-order rate constant $k_{\text {Bile-Gut }}(t)$ could change over time, if the bile flow rate $\left(X_{\text {Bile_Flow }}\right)$ was stimulated. The bile flow rate was described by a turnover model.

$k_{\text {Bile-Gut }}(t)=k_{\text {Bile-Gut, Base }} \cdot X_{\text {Bile_Flow }}$

$\frac{\mathrm{d} X_{\text {Bile_Flow }}}{\mathrm{d} t}=k_{\text {out }} \cdot\left[\left(1+\right.\right.$ Stim $\left.\left._{\text {Bile_Flow }}\right)-X_{\text {Bile_Flow }}\right] \quad$ Initial condition : 1

The production and dissipation of the bile flow $\left(X_{\text {Bile_Flow }}\right)$ were described by a zero-order input rate $\left(K_{\mathrm{in}}=\right.$ $k_{\text {out }}\left(1+\right.$ Stim $\left.\left._{\text {Bile_Flow }}\right)\right)$ and a first-order loss rate constant $\left(k_{\text {out }}\right)$, respectively. As rats lack a gall bladder, they were assumed to have a continuous bile flow that may increase under the effect of food. Therefore, the model included a (small) baseline bile flow rate. The input rate could be stimulated using the variable Stim Bile_Flow which represents the fold increase of bile flow rate. The Stim Bile_Flow $_{\text {was }}$ equal to Smax if the modulus of time past the first dose $\left(\bmod \left(\operatorname{Time}_{\mathrm{dose} 1}, 24 \mathrm{~h}\right)\right)$ was between the time of onset $\left(T_{\mathrm{On}}\right)$ and the time of offset of bile flow stimulation $\left(T_{\text {Off }}\right)$ :

$T_{\text {On }} \leq \bmod \left(\right.$ Time $\left._{\text {dose } 1}, 24 \mathrm{~h}\right)<T_{\text {Off }}$

The Stim Bile_Flow was zero during other times. The mod $\left(\right.$ Time $\left._{\text {dose } 1}, 24 \mathrm{~h}\right)$ is the remainder of Time dose $1_{\text {after }}$ subtracting the largest multiple of $24 \mathrm{~h}$ (i.e., 0, 24, or $48 \mathrm{~h}$, etc.) that is smaller than Time ${ }_{\text {dose } 1 \text {. For example, mod }}$ (Time $_{\text {dose } 1}, 24 \mathrm{~h}$ ) equals $8 \mathrm{~h}$ for Time dose $1=32$ and $5 \mathrm{~h}$ for Time $_{\text {dose } 1}=53 \mathrm{~h}$. This function generates a periodic stimulation of bile flow with a 24-h period length (Fig. 2).

To assure that all $T_{\text {Off }}$ values ranged from $T_{\text {On }}$ to $24 \mathrm{~h}$, $T_{\text {Off }}$ was calculated by $T_{\text {On }}$ plus the fraction $\left(\mathrm{Fr}_{\mathrm{On}}\right)$ of the bile flow stimulating duration to the remaining time $\left(24 \mathrm{~h}-T_{\text {On }}\right)$ as:

$T_{\text {Off }}=T_{\text {On }}+\left(24 \mathrm{~h}-T_{\text {On }}\right) \cdot \mathrm{Fr}_{\mathrm{On}}$

Parameter Variability and Residual Error Model. The BSV was described by a log-normal distribution for all parameters except the fractions that ranged from 0 to 1 and were modeled via a logistic transformation as described previously (30). We utilized allometric scaling for the volume and clearance parameters to scale between rats, dogs, and humans (31). An additive plus proportional residual error model was used for the fimasartan concentrations in each species.

Estimation, Simulation, and Non-compartmental Analysis. Population PK model parameters for all species were simultaneously estimated via the importance sampling algorithm in the S-ADAPT software (version 1.57) $(30,32,33)$. Models were compared using the objective function $(-1 \cdot \log$ likelihood), plausibility of parameter estimates, and standard diagnostic plots for population modeling (11,31,34,35). Simulations were performed using Berkeley Madonna (version
8.3.18) to assess the extent and time-course of EHC. Noncompartmental analysis was performed via the linear trapezoidal method in WinNonlin Professional ${ }^{\mathrm{TM}}$ (version 5.3, Pharsight, Cary, NC).

Impact of EHC. To characterize EHC, the fraction of drug undergoing $\mathrm{EHC}$ and its contribution to the systemic exposure were estimated. The fraction of an oral dose subject to EHC $\left(\mathrm{Fr}_{\mathrm{EHC}}\right)$ was calculated by $\mathrm{Fr}_{\text {Liver }} \cdot \mathrm{Fr}_{\text {Liv-Bile. The }}$ $\mathrm{Fr}_{\text {Liv-Bile }}$ was the fraction of drug that transferred from liver into bile and was subsequently reabsorbed. For the proposed model (Fig. 2), $\mathrm{Fr}_{\text {Liv-Bile }}$ was calculated as $k_{\text {Liv-Bile }} /\left(k_{\text {Liv-Bile }}+\right.$ $\left.k_{\mathrm{el}}\right)$. Plasma concentration vs. time profiles were simulated following oral dosing of fimasartan every $24 \mathrm{~h}$ in humans, dogs, and rats. To evaluate the impact of EHC, plasma concentration profiles without EHC were simulated by removing drug transfer from bile to gut; i.e., drug leaving the bile compartment was assumed to be eliminated instead of entering the gut compartment. Simulations without EHC assumed that bile-cannulation quantitatively collected fimasartan but had no other effect on the PK.

Estimation of Oral Bioavailability. We derived the mathematical solution for the absolute oral bioavailability $\left(F_{\text {oral }}\right)$ for a drug with EHC which accounts for an infinite number of EHC cycles for the proposed model. For dosing of the solution or tablet formulation in the fasting state, the bioavailability without $\mathrm{EHC}$ in our model is $\left(1-\mathrm{Fr}_{\text {liver }}\right)$. After the first EHC cycle, the fraction of dose entering the central compartment is $\mathrm{Fr}_{\mathrm{EHC}} \cdot\left(1-\mathrm{Fr}_{\text {liver }}\right)$. This fraction is $\mathrm{Fr}_{\mathrm{EHC}}$. $\mathrm{Fr}_{\mathrm{EHC}} \cdot\left(1-\mathrm{Fr}_{\text {liver }}\right)$ after two EHC cycles and $\mathrm{Fr}_{\mathrm{EHC}}{ }^{n} \cdot(1-$ $\mathrm{Fr}_{\text {liver }}$ ) after $n$ EHC cycles. Thus, the solution for the total fraction of dose entering the central compartment is the sum over all cycles:

$$
F_{\text {oral }}=\left(1-\mathrm{Fr}_{\text {Liver }}\right) \cdot \sum_{n=0}^{\infty} \operatorname{Fr}_{\mathrm{EHC}}{ }^{n}=\frac{1-\mathrm{Fr}_{\text {Liver }}}{1-\mathrm{Fr}_{\mathrm{EHC}}}=\frac{F_{\text {oral, no EHC }}}{1-\mathrm{Fr}_{\mathrm{EHC}}}
$$

This sum over an infinite number of cycles can be described by the fraction $1 /\left(1-\mathrm{Fr}_{\mathrm{EHC}}\right)$. Thus, the oral bioavailability is $\left(1 /\left(1-\mathrm{Fr}_{\mathrm{EHC}}\right)\right)$-fold larger in the presence of EHC compared with the absence of $\mathrm{EHC}\left(F_{\text {oral, no } \mathrm{EHC}}=\right.$ $\left.1-\mathrm{Fr}_{\text {Liver }}\right)$.

Allometric Scaling to Humans. We examined whether human PK profiles of fimasartan could be predicted from rats and dogs. Allometric scaling to humans was performed using the EHC model based on the models for rats or dogs that were obtained during step 1 of model development (Fig. 1). All parameters for systemic disposition, i.e., $V_{1}, V_{2}, V_{3}, \mathrm{CLd}_{1-}$ Liv, $\mathrm{CLd}_{\text {Shallow, and }} \mathrm{CLd}_{\text {Deep }}$ were scaled from rats $(270 \mathrm{~g}$ body weight) or dogs $(10 \mathrm{~kg})$ to humans $(75 \mathrm{~kg})$ with standard allometric exponents (0.75 for clearances and 1.0 for volumes). Based on the scaled parameters, human plasma concentration profiles after an oral dose of $60 \mathrm{mg}$ fimasartan were predicted based on the rat data (case A) or the dog data (case C).

As the present study provided parameter estimates for bile flow kinetics in humans, we additionally assessed scaling 
by using the human estimates for the bile flow kinetic parameters. For this purpose, we substituted the animal bile flow kinetic parameter estimates (i.e., Smax, $T_{\mathrm{On}}, \mathrm{Fr}_{\mathrm{on}}$, and $k_{\text {out }}$ ) with the population means from humans (rat: case $\mathrm{B}$, or dog: case D; Fig. 1). All simulated cases (A to D) utilized the clearance and volume of distribution estimates from animals. While cases $\mathrm{A}$ and $\mathrm{C}$ used the bile flow kinetic parameters estimated in rats or dogs, cases B and D used the four bile flow kinetic parameter estimates from humans.

The scaled plasma concentrations were simulated at the same time points as those obtained in the human phase I study (21). We then compared the scaled simulated concentrations to the observations in humans. We calculated the apparent terminal half-life $\left(\mathrm{t}_{1 / 2}\right)$, as well as the areas under the plasma concentration time curve from 0 to $24 \mathrm{~h}\left(\mathrm{AUC}_{0-24 \mathrm{~h}}\right)$ and from $0 \mathrm{~h}$ to infinity $\left(\mathrm{AUC}_{0-\infty}\right)$ via non-compartmental analysis. The scaled $\mathrm{AUC}_{0-24} \mathrm{~h}, \mathrm{AUC}_{0-\infty}$ and $t_{1 / 2}$ for cases $\mathrm{A}$ to $\mathrm{D}$ were compared with the non-compartmental parameter estimates for the phase I study (21). Additionally, to compare the scaled predicted and observed concentration profiles over time (36), we calculated the mean relative prediction error (MPE) as a measure of bias:

MPE $=\frac{1}{N} \sum_{1}^{N} \frac{C_{\text {pred, } \mathrm{i}}-C_{\text {obs, } \mathrm{i}}}{C_{\text {obs, } \mathrm{i}}}$

The $C_{\text {pred, } i \mathrm{i}}$ is the predicted concentration and $C_{\mathrm{obs}, \mathrm{i}}$ the mean observed concentration at the $i$ th time point and $N$ is the total number of plasma concentrations for each profile.

\section{RESULTS}

Systemic Disposition. To describe the systemic disposition of fimasartan, a three-compartment model consisting of a central, a shallow peripheral, and a deep peripheral compartment was superior to a two-compartment model for the rat data (improvement in $-2 \cdot \log$-likelihood, $91.1 ; p<0.0001)$ and thus chosen as final model (Fig. 2). Without a deep peripheral compartment, low plasma concentrations in rats could not be fitted.

We initially modeled the rat, dog, and human data separately (Fig. 1) using the same model structure and found that most systemic disposition parameters $\left(V_{1}, V_{2}, \mathrm{CLd}_{\text {Shallow, }}\right.$ and $\mathrm{CLd}_{\text {Deep }}$ ) could be scaled well allometrically (Table I). However, $V_{3}$ and $\mathrm{CLd}_{1-\text { liv }}$ differed between species and were thus modeled with separate estimates for each species. Parameters for oral absorption and EHC were estimated separately for each species without scaling. This provided the best curve fits and allowed us to assess differences in the extent and time-course of EHC between rats, dogs, and humans (Figs. 3, 4, and 5; Figs. S1, S2, S3, and S4).

Absorption. The mean dissolution half-life for the capsule and tablet formulations was $22.4 \mathrm{~min}$ in dogs, but the capsule had a lower relative oral bioavailability compared with the tablet ( $F_{\text {cap }}, 57.2 \%$, Table I). In humans, dissolution/ disintegration was slower for the 20- and 60-mg tablets compared with higher doses $\left(k_{\mathrm{dis}}\right.$, Table I). The absorption half-life $\left(\ln (2) / k_{\text {abs }}: 1.16 \mathrm{~min}\right)$ of dissolved fimasartan from the gut into the central or liver compartment was short in all species, suggesting rapid absorption after dissolution.

The decreased relative bioavailability in the fed state $\left(F_{\text {Food, Rel }}\right)$ and for the capsule $\left(F_{\text {Cap }}\right)$ was modeled as drug loss during transfer from the stomach to the gut compartment. The model described the fraction of absorbed drug entering the central compartment without any metabolism as $\left(1-\mathrm{Fr}_{\text {liver }}\right)$ (Fig. 2). This fraction is equal to the product of the fraction absorbed $\left(F_{\mathrm{a}}\right)$, the fraction of drug not metabolized in the gut wall $\left(F_{\mathrm{g}}\right)$, and the fraction of drug escaping the liver during the first pass $\left(F_{\mathrm{h}}\right)$. Estimation of the parameter $\mathrm{Fr}_{\text {Liver }}$ allowed us to simplify the model structure. The $\mathrm{Fr}_{\text {Liver }}$ is the fraction of drug in the gut compartment which enters the liver compartment instead of the systemic circulation (i.e., com-

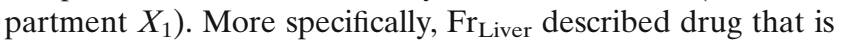
to be non-absorbed, metabolized in the gut wall or liver, or secreted into bile with subsequent reabsorption from the gut. Inclusion of $\mathrm{Fr}_{\text {Liver }}$ significantly improved the curve fits $(p<0.0001$, likelihood ratio test).

Fimasartan in the liver compartment was subject to firstorder elimination by various process $\left(k_{\mathrm{el}}\right)$ and secretion into bile ( $\left.k_{\text {Liv-Bile }}\right)$ (Fig. 2). In the model, elimination from the liver compartment $\left(k_{\mathrm{el}}\right)$ represented all types of drug loss before the systemic circulation, including unabsorbed drug, drug metabolized in the gut wall or liver, and drug eliminated into bile in a form that is not reabsorbed. The $k_{\mathrm{el}}$ did however not represent biliary secretion of metabolite(s) (such as glucuronides) that are reverted to fimasartan and reabsorbed. In the model, all drug (i.e., fimasartan and any reversible metabolites) in the bile compartment were assumed to be reabsorbed. The concentrations of reversible metabolite(s) such as the glucuronide were not quantifiable in plasma, feces, and urine (16); this made it not feasible to include reversible metabolites explicitly in the model. Thus, any metabolite that undergoes reversible metabolism and is subsequently reabsorbed was regarded as fimasartan in the model.

Fimasartan transferred from the central compartment into the liver compartment. The reverse process, from the liver into the central compartment, was considered, but the associated clearance was over 500-fold smaller than $\mathrm{CLd}_{1-\mathrm{Liv}}$. As inclusion of clearance from the liver to the central compartment neither improved the curve fits nor the objective function, this process was not included in the final model (Fig. 2). This model described the extent and time-course of EHC excellently in all species (Figs. 3, 4, and 5; Figs. S1, S2, S3, and S4) and could capture multiple (i.e., >2) peaks after a single dose (Fig. 3) and EHC after multiple dosing (Fig. 5; Fig. S3). The observed vs. individual or population fitted concentrations were unbiased and reasonably precise in all species (Fig. S4). The normalized prediction distribution errors (Fig. S4) and visual predictive checks (not shown) for each species, formulation, and dose revealed an adequate predictive performance.

Impact of EHC. The final model enabled quantitative comparisons of EHC among species. The median estimated $\mathrm{Fr}_{\text {liver }}$ ranged from $91 \%$ to $92 \%$ in all species (Table II), suggesting that orally dosed fimasartan undergoes a significant first-pass uptake into the liver. The individual $\mathrm{Fr}_{\mathrm{EHC}}$ ranged from $56.5 \%$ to $88.7 \%$ in rats and humans but were less than $46 \%$ in dogs indicating less extensive $\mathrm{EHC}$ in dogs (Table II). This was in good agreement with the smaller 


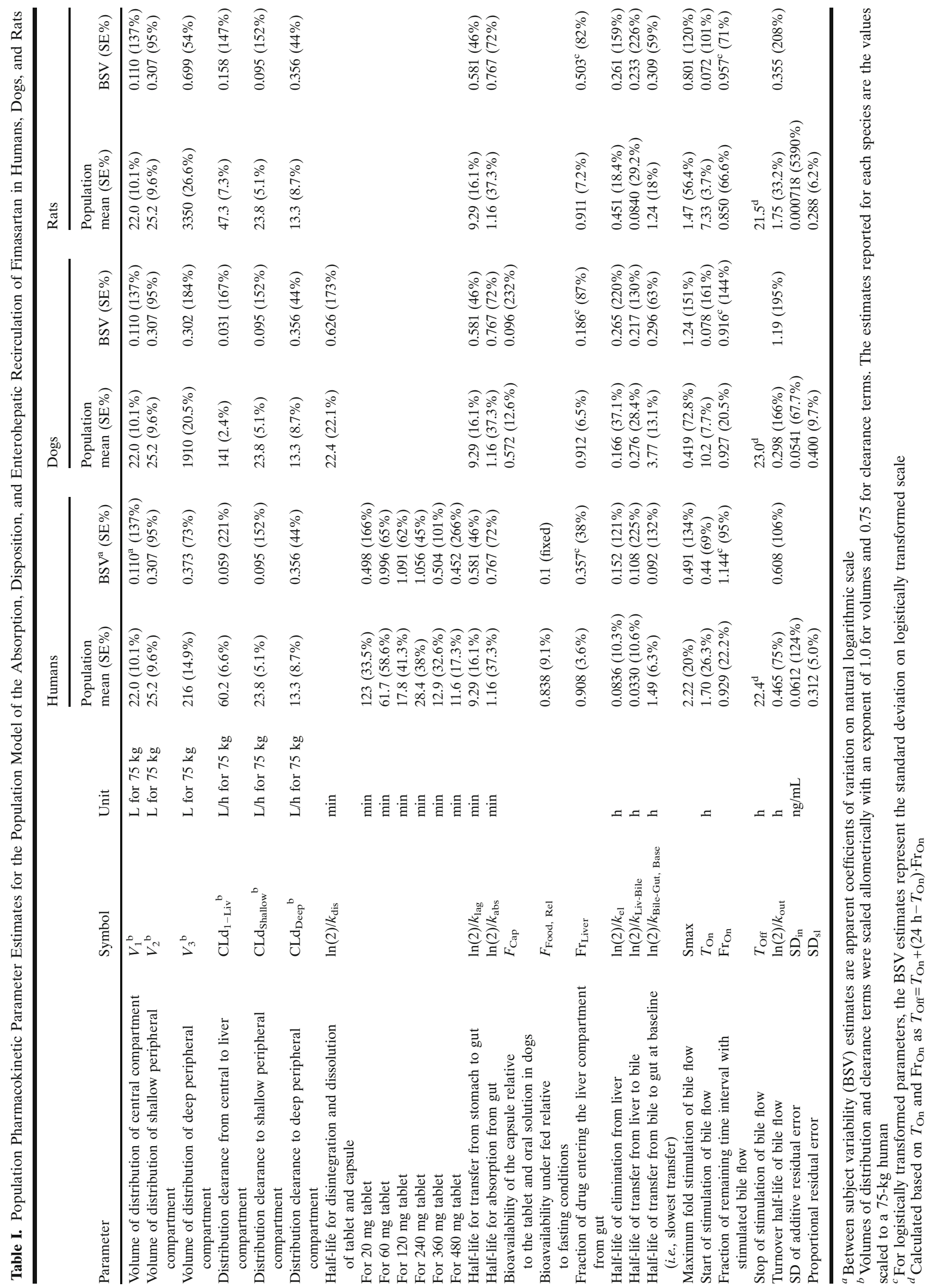


Rat (log scale)

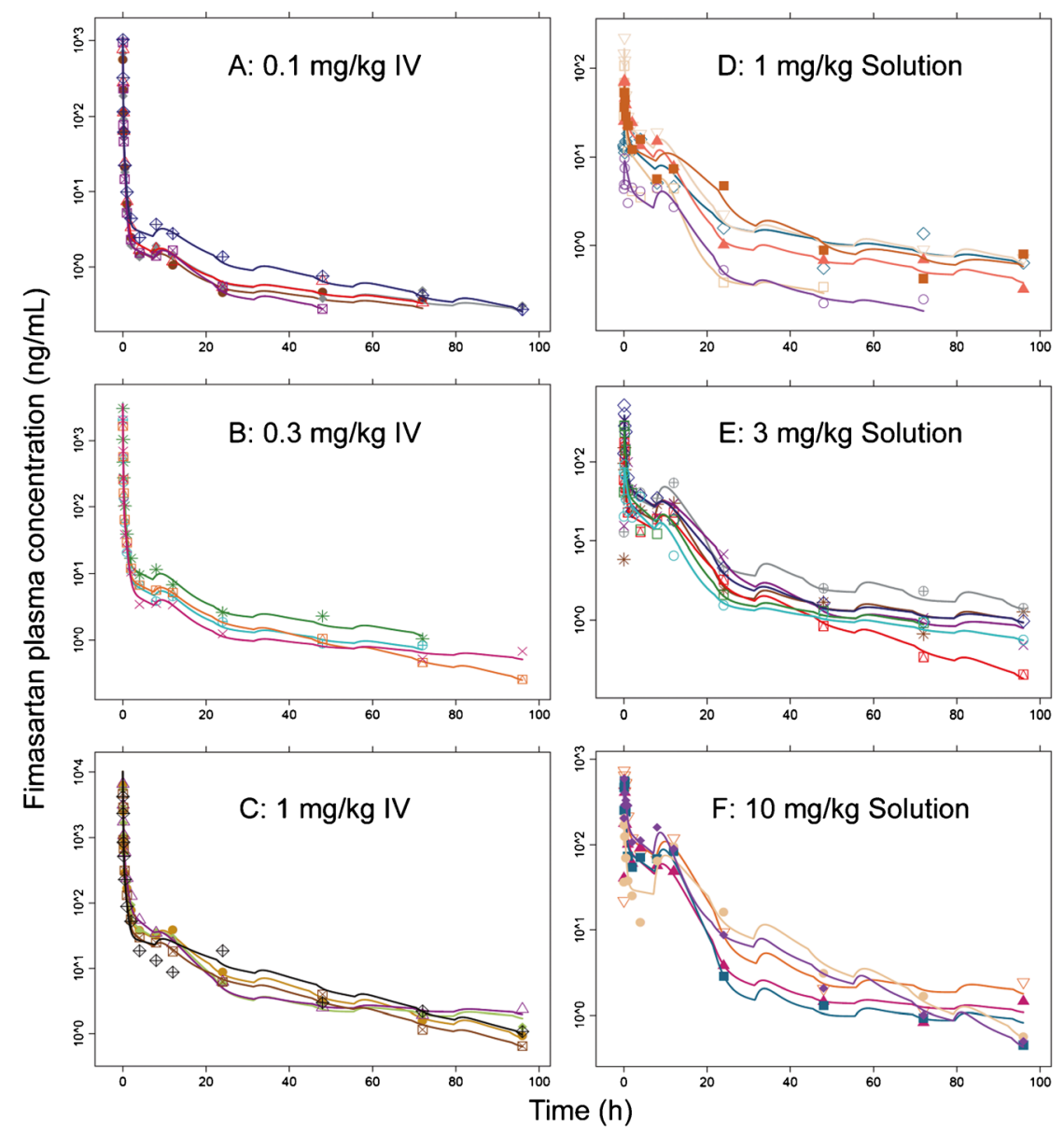

Fig. 3. Observed and individual fitted plasma concentrations of fimasartan in rats after a single intravenous or oral dose at different dose levels on logarithmic scale

second peaks in dogs compared with rats and humans (Figs. 3, 4, and 5; Figs. S1, S2, and S3). Second peaks and "shoulders" were observed for IV and oral dosing demonstrating the presence of EHC. Second peaks were smaller after IV compared with oral dosing. The model explained this by the large estimates for $\mathrm{Fr}_{\text {liver }}$.

Oral bioavailability and the area under the plasma concentration-time curve (AUC) after oral dosing of fimasartan with EHC divided by the AUC without EHC was highest in rats and lowest in dogs (Fig. 6). After a single dose, simulations over $1000 \mathrm{~h}$ predicted that the fraction of dose entering the bile compartment was $537 \%$ in normal and $84.3 \%$ in bile duct-cannulated rats, $60 \%$ in normal and $38 \%$ in bile duct-cannulated dogs, and $253 \%$ in normal and $72 \%$ in bile flow shunted humans. In the absence of bile duct cannulation, predicted fractions larger than $100 \%$ indicate that fimasartan molecules (including reversible metabolites) underwent on average more than one EHC cycle.

Scaling to Human. Allometrically scaled predictions from rats to humans (cases $\mathrm{A}$ and $\mathrm{B}$ ) and from dogs to humans (cases $\mathrm{C}$ and $\mathrm{D}$ ) are shown in Fig. 7. When the bile flow kinetic parameters (i.e., Smax, $T_{\mathrm{On}}, \mathrm{Fr}_{\mathrm{on}}$, and $k_{\text {out }}$ ) in humans were combined with the allometrically scaled clearance and volume of distribution estimates from rats (case B), the timing of the second peak due to EHC was better captured compared with case A (Fig. 7a). The AUC predictions for scaling from rats to humans (cases A and B, Table III) matched the observed AUC better compared with scaling from dogs to humans (cases C and D, Table III, Fig. 7b). This was in agreement with the substantially smaller extent of EHC in dogs (Table II) and the 2.3-fold higher allometrically scaled clearance $\left(\mathrm{CLd}_{1-\mathrm{Liv}}\right)$ in dogs compared with those in humans (Table I). Both of these factors led to biased predictions of human PK profiles in the presence of EHC based on the dog data (Fig. 7b). For rat-to-human scaling, bias was $10 \%$ or less (Table III), whereas dog-to-human scaling yielded a bias of approximately $-80 \%$ (cases C and D).

Oral Bioavailability. The calculated absolute bioavailability of fimasartan (Eq. 13, Table II) matched the noncompartmental absolute bioavailability estimates in rats (16) and dogs well. In the absence of absolute oral bioavailability data for fimasartan in humans, the model allowed us to predict the absolute oral bioavailability in humans based on the individual PK parameter estimates of the final model (Table II). The relative oral bioavailability in the fed state was slightly lower than that in the fasting state $\left(F_{\mathrm{Food}, \mathrm{Rel}}, 83.8 \%\right.$, Table I) similar to a previous report (37). Bioavailability of the tablet and solution were similar and significantly higher than bioavailability of the capsule in dogs $\left(F_{\text {cap }}\right.$, Table I). 
Dog (log scale)
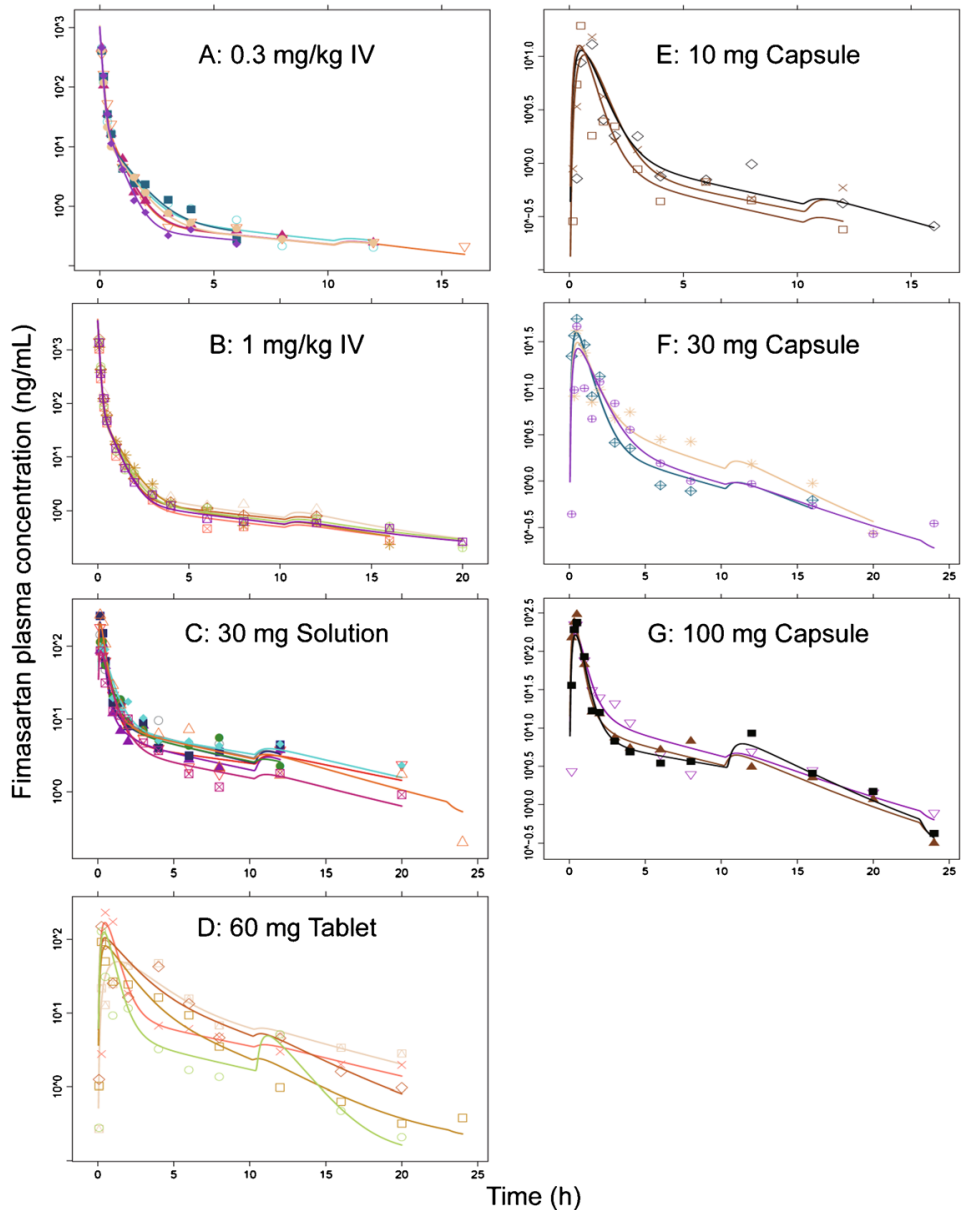

Fig. 4. Observed and individual fitted plasma concentrations of fimasartan in dogs after a single intravenous or oral dose at different dose levels on logarithmic scale

\section{DISCUSSION}

Enterohepatic recirculation can have a pronounced impact on the systemic drug exposure and thus on the pharmacological effects (8). Conceptually, the extent of EHC is determined by the fraction of drug which is excreted from the liver via bile into the gut and subsequently reabsorbed from the gut into the systemic circulation and the liver. This circulation can involve reversible formation of glucuronide metabolites. While the extent of EHC can be assessed experimentally by bile duct cannulation, this approach has several important limitations including disruption of the physiology and the requirement of complicated experimental procedures which hamper translation to humans. Recently, human biliary clearance was predicted for drugs undergoing EHC based on in vitro systems (such as sandwich cultured human hepatocytes) (38). Nevertheless, the prediction of human PK for drugs undergoing EHC is not straightforward due to limited knowledge about the expression of hepatobiliary transporter(s) across species. Furthermore, there are considerable differences in the glucuronidase activity between rats, dogs and humans (39) and rats do not have a gall bladder. However, rats may have an increased bile flow rate due to food. Therefore, the extent of EHC may differ considerably between species and may hamper inter-species scaling for drugs with a significant extent of EHC.

Population PK modeling can characterize the impact of EHC on bioavailability and plasma concentration-time profiles. A quantitative understanding and population model for the extent and time-course of EHC in relevant species in view of potential inter-species differences could be valuable for drug development.

Various models for EHC have been developed $(8,24-$ 28,40-60), including physiologically based PK models with EHC (61-63). These models either assumed continuous enterohepatic recirculation (25,40-42), one or multiple secretions of bile using an on/off switch (i.e., "lag-time(s)") $(24,26,43-58)$ or implemented a sine function to describe periodic bile releases $(27,28,59,60)$. We are not aware of published models that simultaneously quantified EHC across more than one species or described the full time-course of PK profiles after multiple dosing. Thus, it is not well known how to translate the extent and time-course of EHC across species.

We developed the first population model that simultaneously described the extent and time-course of EHC in multiple 


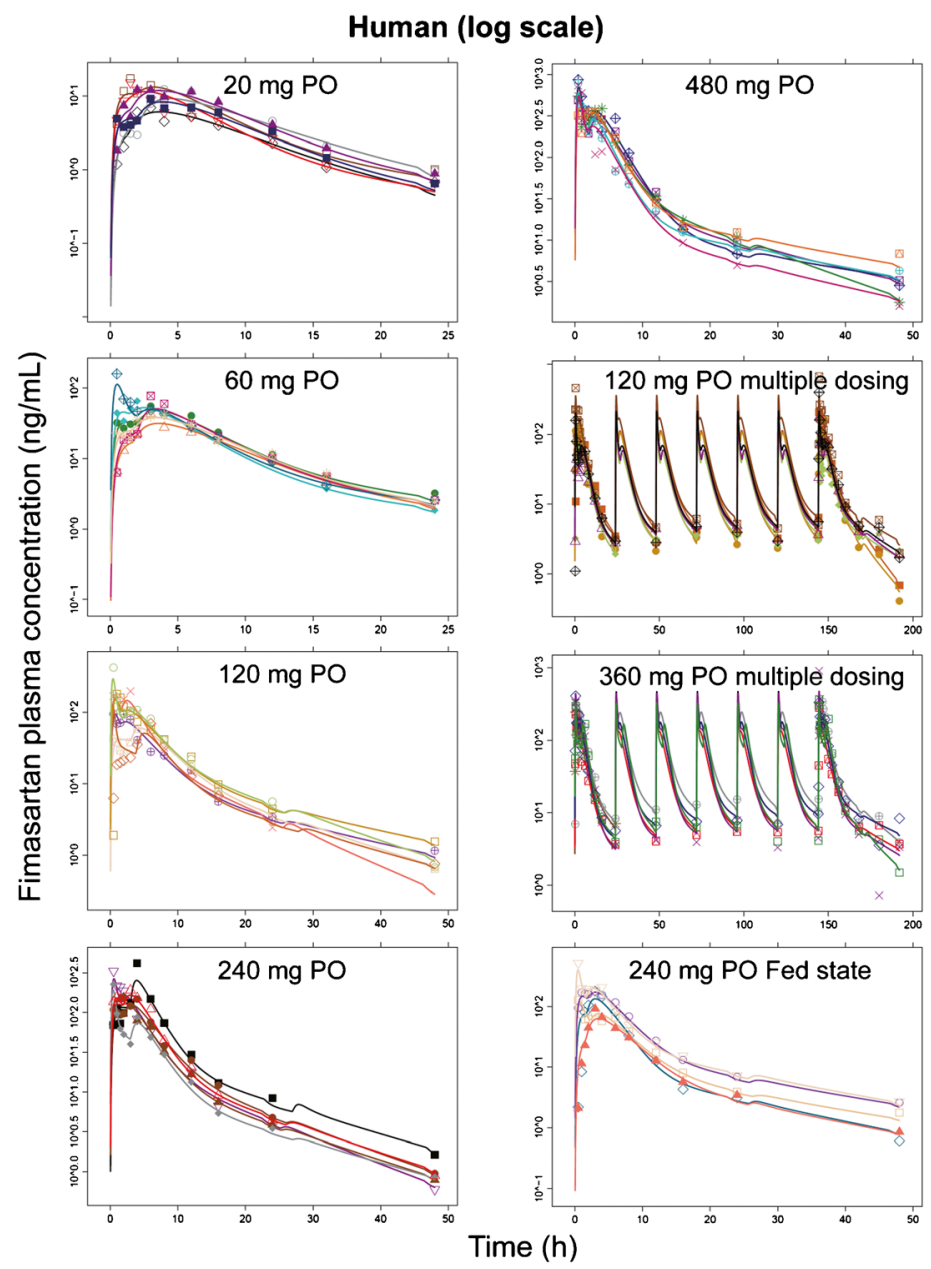

Fig. 5. Observed and individual fitted plasma concentrations of fimasartan in healthy volunteers after a single or multiple oral doses at different dose levels on logarithmic scale

species (rats, dogs, and humans) after IV and single or multiple oral dosing. This model contained 45 structural parameters that were simultaneously estimated with adequate precision (relative standard errors below $38 \%$ for 38 of 45 population means, Table I). During the development of fimasartan, animal studies showed the occurrence of multiple peaks after IV and oral dosing. As second peaks were also observed after IV dosing, they were likely caused by EHC. We applied population modeling to account for BSV and simultaneously describe EHC of fimasartan across multiple species.

After oral administration, drugs are absorbed through the gastrointestinal membrane and can be metabolized while passing through enterocytes and the liver (i.e., a first-pass effect). The UDP-glucuronosyltransferase (UGT) is responsible for glucuronidation and is widely expressed in the liver and gastrointestinal tract (64-66). Morphine, fenoterol, and ARBs such as losartan are extensively glucuronidated in the small intestine $(67,68)$. In our previous study (16), we observed that $11.8 \%$ of the total fimasartan dose was recovered in bile as a glucuronide conjugate in bile ductcannulated rats. We did not model data from bile ductcannulated rats due to a potential disturbance of the physiology by cannulation.
Significant first-pass metabolism of fimasartan would result in larger second peaks after oral compared with IV dosing. This was observed in our data and well captured by the proposed model. Modeling indicated that the fraction ( Fr $\left._{\mathrm{EHC}}\right)$ of drug undergoing enterohepatic recirculation was similar in rats and humans and smaller in dogs (Table II). Inclusion of a direct absorption process from the gut into the liver compartment $\left(\mathrm{Fr}_{\text {liver }}\right.$; i.e., a first-pass effect) provided significantly better curve fits $(p<0.0001)$ indicating a considerable contribution of the first-pass effect. This mechanism explained why the second peaks were much more pronounced after oral compared with IV dosing.

Plasma fimasartan exposure was substantially higher in the presence compared with the absence of EHC in humans and rats (Fig. 6). In our model, elimination from the liver includes metabolism of fimasartan as well as biliary elimination of fimasartan and any metabolites that are not reabsorbed from the gut. Fimasartan as parent and its glucuronide metabolite are the most prevalent species in bile (16), but the glucuronide metabolite is not found in feces, urine, and plasma. We did not model reversible metabolism to maintain a simpler model structure as we described previously $(69,70)$. 
Table II. Fraction of Fimasartan Transferring from Liver into Bile and Undergoing Enterohepatic Recirculation and Calculated Absolute Bioavailability

\begin{tabular}{|c|c|c|c|}
\hline & Humans & Dogs & Rats \\
\hline $\begin{array}{l}\text { Fraction of fimasartan transferring from } \\
\text { gut into the liver compartment }\left(\mathrm{Fr}_{\text {Liver }}\right)\end{array}$ & $91.3 \%(80.9-95.0 \%)$ & $91.4 \%(89.0-93.5 \%)$ & $91.7 \%(80.2-96.9 \%)$ \\
\hline $\begin{array}{l}\text { Fraction of fimasartan in liver that undergoes } \\
\text { EHC and is reabsorbed }\left(\mathrm{Fr}_{\text {Liver-Bile }}\right)\end{array}$ & $72.1 \%(63.8-76.8 \%)$ & $36.8 \%(25.7-50.6 \%)$ & $83.7 \%(77.4-91.5 \%)$ \\
\hline $\begin{array}{l}\text { Fraction of fimasartan that enters the liver } \\
\text { from gut and is subsequently reabsorbed } \\
\left(\operatorname{Fr}_{\mathrm{EHC}}=\mathrm{Fr}_{\text {Liver }} \cdot \operatorname{Fr}_{\text {Liver-Bile }}\right)\end{array}$ & $65.6 \%(56.5-72.0 \%)$ & $33.3 \%(24.0-45.9 \%)$ & $76.3 \%(64.9-88.7 \%)$ \\
\hline Modeled absolute oral bioavailability & $\begin{array}{l}\text { Tablet fasting }(n=42), 25.1 \% \\
\quad(15.1-43.9 \%) . \text { Tablet fed } \\
(n=5)^{\mathrm{a}}, 18.2 \%(12.2-31.0 \%)\end{array}$ & $\begin{array}{l}\text { Solution, } 15.4 \%(11.0-17.6 \%) \\
\text { Tablet, } 13.9 \%(8.56-16.0 \%) \\
\text { Capsule, } 7.13 \%(6.31-8.35 \%)\end{array}$ & $\begin{array}{r}\text { Solution, } 38.7 \% \\
\quad(20.0-59.8 \%)\end{array}$ \\
\hline $\begin{array}{l}\text { Observed absolute oral bioavailability } \\
\text { by non-compartmental analysis }\end{array}$ & & $\begin{array}{l}\text { Solution, } 14.6 \%(8.01-17.3 \%) \\
\text { Tablet, } 10.2 \%(3.90-12.8 \%) \\
\text { Capsule, } 5.95 \% \\
(4.04-6.83 \%)\end{array}$ & $\begin{array}{l}\text { Solution, } 41.1 \%{ }^{b} \\
\quad(15.1-73.2 \%)\end{array}$ \\
\hline
\end{tabular}

The values shown are the medians (ranges) based on the individual pk parameter estimates from the final population pk analysis. The medians closely matched the typical values calculated using the population means

${ }^{a}$ The estimated relative bioavailability in the fed $v s$. the fasting state was $83.8 \%$ for the $240 \mathrm{mg}$ oral dose in humans

${ }^{b}$ The absolute bioavailability data for the oral solution was taken from Kim et al. (16)

The extent of EHC was similar in rats and humans and smaller in dogs; however, the time-course of EHC differed between rats and humans (Table I). The developed model described the time-course of EHC by a turnover process for bile flow (Fig. 2). Baseline bile flow was normalized to $100 \%$ and bile flow was stimulated between times $T_{\text {On }}$ and $T_{\text {Off }}$ with a maximum fold increase (Smax). The rates of increase and decrease of bile flow over time were captured by the turnover half-life $(\ln (2) /$ $\left.k_{\text {out }}\right)$. While bile flow could change rapidly in humans (half-life, $0.465 \mathrm{~h}$ ) and $\operatorname{dogs}(0.298 \mathrm{~h})$, the turnover halflife in rats was longer $(1.75 \mathrm{~h})$ possibly due to rats lacking a gall bladder. The maximum stimulation of bile flow was largest in humans (Smax, 2.22) and smaller in dogs and rats. The extensive stimulation and rapid turnover of bile flow in humans caused pronounced second peaks (Fig. 5; Fig. S3). Importantly, Smax and the turnover half-life of bile flow only affect the shape of the plasma concentration time curves but not the extent of EHC. The use of a turnover model for bile flow allowed us to excellently capture the timing and shape of multiple peaks (Figs. 3, 4, and 5) across three species. This approach can implement multiple stimulations of bile flow (e.g., due to food intake) and describe the time-course of bile flow over multiple days (Fig. 5).

Human PK profiles in the presence of EHC were predicted by this allometrically scaled model based on the rat data (Fig. 7; Table III). It was beneficial to combine the clearances and volumes of distribution from rats with the estimated bile flow kinetic parameters in humans (case B vs. case A; Figs. 1 and 7). Future studies will have to show, whether the bile flow kinetic parameters for fimasartan in humans (used in cases B and D) are transferrable to other drugs. The EHC was much less pronounced and $\mathrm{CLd}_{1-\mathrm{Liv}}$ was 2.3-fold higher in dogs compared with humans. Therefore, the human PK predictions based on the dog data were

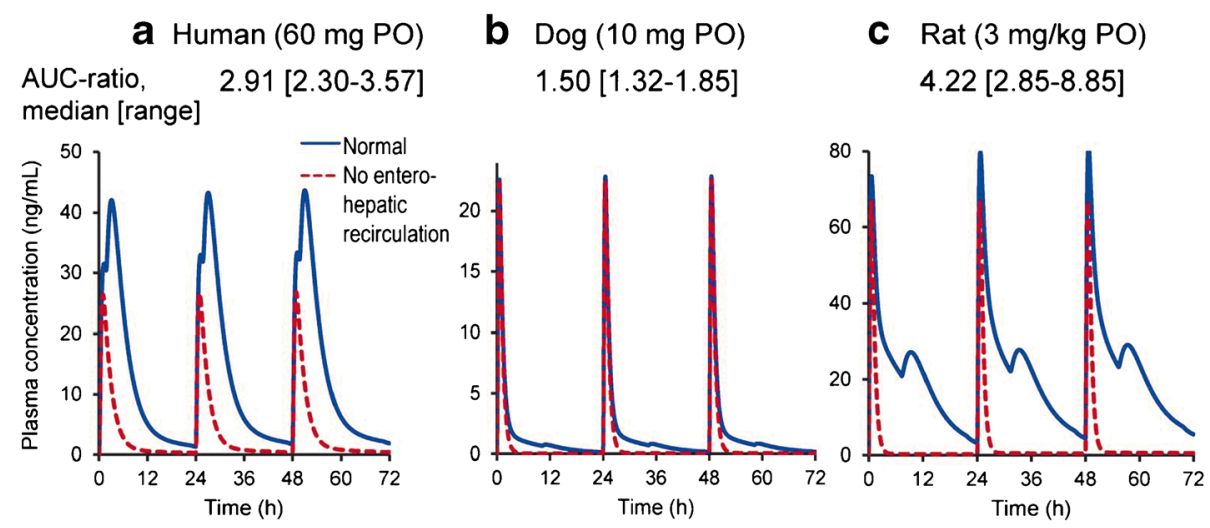

Fig. 6. Simulated concentrations after oral dosing of fimasartan every $24 \mathrm{~h}$ in humans, dogs, and rats. The dashed lines refer to healthy volunteers, dogs and rats that had no enterohepatic recirculation of fimasartan (i.e., in the model, all drug leaving the bile compartment was assumed to be eliminated instead of entering the gut compartment). The area under the curve (AUC) ratio describes the relative drug exposure in the presence compared with the absence of EHC 

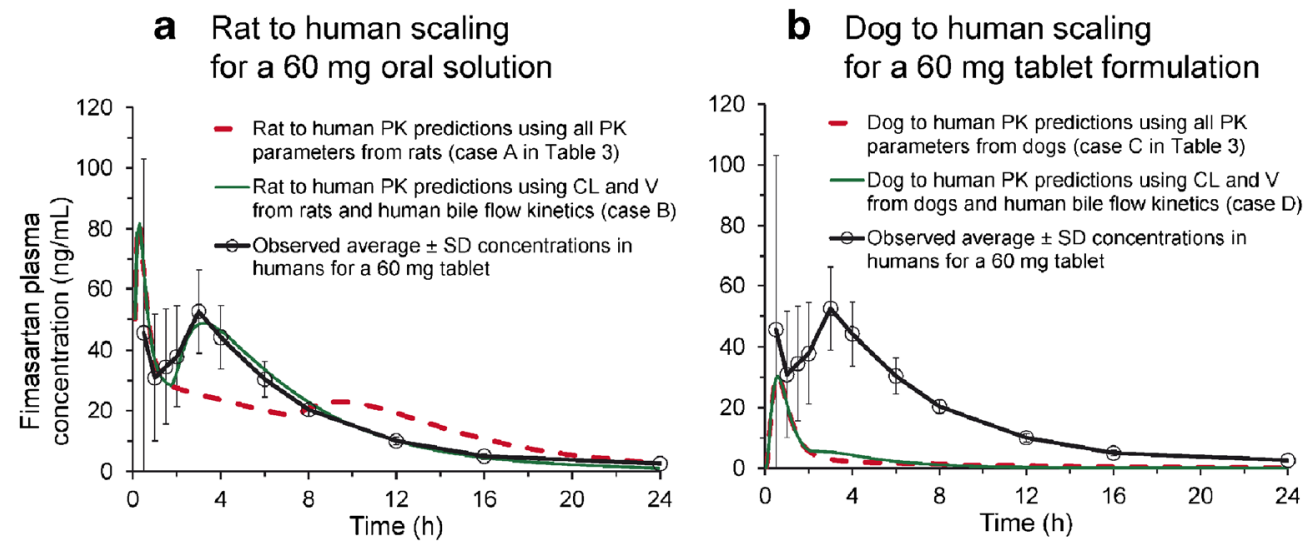

Fig. 7. Predicted plasma concentrations for an oral solution of $60 \mathrm{mg}$ fimasartan based on the rat model or a $60-\mathrm{mg}$ tablet based on the dog model. The allometrically scaled estimates from animals for volumes of distribution $\left(V_{1}, V_{2}\right.$, and $\left.V_{3}\right)$ and clearances $\left(\mathrm{CL}_{1-\mathrm{Liv}}, \mathrm{CLd}_{\text {Shallow }}\right.$, and $\left.\mathrm{CLd}_{\text {Deep }}\right)$ were used for all scaled human predictions (solid and broken lines). The broken lines represent scaled human predictions from rats (case A) or dogs (case C) using all PK parameter estimates from animals. In contrast, the solid lines represent the scaled human predictions from rats (case B) or dogs (case D) using the human bile flow kinetic parameter estimates (i.e., $T_{\mathrm{On}}$, Smax, $\mathrm{Fr}_{\mathrm{On}}$ and $k_{\text {out }}$; Table I). None of these lines represent curve fits

biased and inferior to the translation from rats to humans (Fig. 7; Table III). Future studies are required to explore whether predictions from rats to humans are better than those from dogs to humans for other drugs undergoing EHC. To our knowledge, this study presents the first attempt of scaling the extent and time-course of EHC from animals to humans.

Repeated reabsorption due to EHC may affect oral bioavailability. We derived the equation for the absolute oral bioavailability. This solution for our model was identical to the mathematically proven mass balance solutions for more complex models containing EHC $(69,70)$ supporting that our model simplifications were adequate. Our model-based estimates for the absolute oral bioavailability in rats and dogs were well comparable with the estimates determined via non-compartmental analysis (Table II). This demonstrated that the model adequately described oral bioavailability, although we made the modeling assumption that all drug entering the bile is reabsorbed. The higher bioavailability of fimasartan for the tablet compared with the capsule formulation supported the choice to formulate fimasartan as a tablet for use in patients. Finally, modeling enabled us to predict the absolute oral bioavailability with a median of $25.1 \%$ in the fasting and $18.2 \%$ in the fed state for fimasartan in humans (Table II). These predictions, in the absence of IV data in humans, are to be confirmed by future clinical studies.

In summary, we developed a population PK model that simultaneously described the extent and timecourse of EHC in rats, dogs, and humans. This model yielded adequate curve fits and highlighted the extensive EHC for fimasartan in rats and humans and a smaller extent of EHC in dogs. The proposed model could describe multiple peaks after single and multiple doses and holds promise to support the characterization of $\mathrm{EHC}$ and rational dose selection for fimasartan in humans.

Table III. Observed Pharmacokinetic Parameters for a $60 \mathrm{mg}$ Fimasartan Tablet in Humans and Scaled Pharmacokinetic Parameters from Rats to Humans (Cases A and B, for a 60-mg Solution) and from Dogs to Humans (Cases C and D, for a 60-mg Tablet)

\begin{tabular}{|c|c|c|c|c|}
\hline Case & $\begin{array}{l}\mathrm{AUC}_{0-24 \mathrm{~h}} \\
(\mathrm{ng} \cdot \mathrm{h} / \mathrm{mL})\end{array}$ & $\begin{array}{l}\mathrm{AUC}_{0-\infty} \\
(\mathrm{ng} \cdot \mathrm{h} / \mathrm{mL})\end{array}$ & $t_{1 / 2}(\mathrm{~h})$ & Bias $(\%)$ \\
\hline$\overline{\text { Average } \pm \text { SD in humans (observed) }}$ & $405 \pm 64$ & $424 \pm 63$ & $5.10 \pm 0.76$ & \\
\hline A. Scaling from rats to humans (all PK parameters from rats) & 403 & 420 & 4.30 & $10 \%$ \\
\hline $\begin{array}{l}\text { B. Scaling from rats to humans (CL and } \mathrm{V} \text { from rats and bile } \\
\text { flow kinetics from humans) }\end{array}$ & 416 & 421 & 3.55 & $-1 \%$ \\
\hline C. Scaling from dogs to humans (all PK parameters from dogs) & 55.6 & 56.6 & 4.71 & $-79 \%$ \\
\hline $\begin{array}{l}\text { D. Scaling from dogs to humans (CL and V from dogs and bile } \\
\text { flow kinetics from humans) }\end{array}$ & 56.7 & 56.8 & 2.57 & $-80 \%$ \\
\hline
\end{tabular}

${ }^{a}$ Bias represents the mean relative prediction error of the fimasartan plasma concentrations at the time points observed in the clinical study (formula provided in the "MATERIALS AND METHODS"). The optimal bias value is $0 \%$ 


\section{ACKNOWLEDGMENTS}

This study was supported by a grant of the Korean Health Technology R\&D Project, Ministry of Health and Welfare, Republic of Korea (HI13C1130). CBL and JBB are the recipients of Career Development Fellowships by the Australian National Health and Medical Research Council (NHMRC, fellowship: 1062509 to CBL and 1084163 to JBB).

Conflict of Interest All authors declare no conflict of interest.

\section{REFERENCES}

1. Christ DD, Wong PC, Wong YN, Hart SD, Quon CY, Lam GN. The pharmacokinetics and pharmacodynamics of the angiotensin II receptor antagonist losartan potassium (DuP 753/MK 954) in the dog. J Pharmacol Exp Ther. 1994;268(3):1199-205.

2. Miura M, Satoh S, Inoue K, Saito M, Habuchi T, Suzuki T. Telmisartan pharmacokinetics in Japanese renal transplant recipients. Clin Chim Acta Int J Clin Chem. 2009;399(1-2):83-7.

3. Ieiri I, Nishimura C, Maeda K, Sasaki T, Kimura M, Chiyoda T, et al. Pharmacokinetic and pharmacogenomic profiles of telmisartan after the oral microdose and therapeutic dose. Pharmacogenet Genomics. 2011;21(8):495-505.

4. Davi H, Tronquet C, Miscoria G, Perrier L, DuPont P, Caix J, et al. Disposition of irbesartan, an angiotensin II AT1-receptor antagonist, in mice, rats, rabbits, and macaques. Drug Metab Dispos Biol Fate Chem. 2000;28(1):79-88.

5. Deguchi T, Watanabe N, Kurihara A, Igeta K, Ikenaga H, Fusegawa K, et al. Human pharmacokinetic prediction of UDPglucuronosyltransferase substrates with an animal scale-up approach. Drug Metab Dispos Biol Fate Chem. 2011;39(5):8209.

6. Dobrinska MR. Enterohepatic circulation of drugs. J Clin Pharmacol. 1989;29(7):577-80.

7. Sorgel F, Kinzig M. Pharmacokinetics of gyrase inhibitors, part 2: renal and hepatic elimination pathways and drug interactions. Am J Med. 1993;94(3A):56S-69S.

8. Roberts MS, Magnusson BM, Burczynski FJ, Weiss M. Enterohepatic circulation: physiological, pharmacokinetic and clinical implications. Clin Pharmacokinet. 2002;41(10):751-90.

9. Bulitta JB, Landersdorfer CB, Kinzig M, Holzgrabe U, Sorgel F. New semiphysiological absorption model to assess the pharmacodynamic profile of cefuroxime axetil using nonparametric and parametric population pharmacokinetics. Antimicrob Agents Chemother. 2009;53(8):3462-71.

10. Holford NH, Ambros RJ, Stoeckel K. Models for describing absorption rate and estimating extent of bioavailability: application to cefetamet pivoxil. J Pharmacokinet Biopharm. 1992;20(5):421-42.

11. Bulitta JB, Okusanya OO, Forrest A, Bhavnani SM, Clark K, Still JG, et al. Population pharmacokinetics of fusidic acid: rationale for front-loaded dosing regimens due to autoinhibition of clearance. Antimicrob Agents Chemother. 2013;57(1):498507.

12. Ogungbenro K, Pertinez H, Aarons L. Empirical and semimechanistic modelling of double-peaked pharmacokinetic profile phenomenon due to gastric emptying. AAPS J. 2015;17(1):22736.

13. Kim TW, Yoo BW, Lee JK, Kim JH, Lee KT, Chi YH, et al. Synthesis and antihypertensive activity of pyrimidin-4(3H)-one derivatives as losartan analogue for new angiotensin II receptor type 1 (AT1) antagonists. Bioorg Med Chem Lett. 2012;22(4):1649-54.

14. Lee SE, Kim YJ, Lee HY, Yang HM, Park CG, Kim JJ, et al. Efficacy and tolerability of fimasartan, a new angiotensin receptor blocker, compared with losartan $(50 / 100 \mathrm{mg})$ : a $12-$ week, phase III, multicenter, prospective, randomized, double- blind, parallel-group, dose escalation clinical trial with an optional 12-week extension phase in adult Korean patients with mild-to-moderate hypertension. Clin Ther. 2012;34(3):552-68-68 e1-9.

15. Yi S, Kim TE, Yoon SH, Cho JY, Shin SG, Jang IJ, et al. Pharmacokinetic interaction of fimasartan, a new angiotensin II receptor antagonist, with amlodipine in healthy volunteers. J Cardiovasc Pharmacol. 2011;57(6):682-9.

16. Kim TH, Shin S, Bashir M, Chi YH, Paik SH, Lee JH, et al. Pharmacokinetics and metabolite profiling of fimasartan, a novel antihypertensive agent, in rats. Xenobiotica. 2014;44(10):913-25.

17. Lane A, Engmann E, Bryson S, Lee J, Tan H, Chi Y. Single dose pharmacokinetics (PK) and pharmacodynamics (PD) of BR-A657, an angiotensin II (AII) antagonist. Clin Pharmacol Ther. 2005;77(2):P59-P.

18. Lane A, Kleinermanns D, Bryson S, Lee J, Tan H, Chi Y. Multiple dose pharmacokinetics (PK) and pharmacodynamics (PD) of BR-A-657, an angiotensin II (AII) antagonist. Clin Pharmacol Ther. 2005;77(2):P58-P.

19. Zhou H. Pharmacokinetic strategies in deciphering atypical drug absorption profiles. J Clin Pharmacol. 2003;43(3):211-27.

20. Jeon H, Lim KS, Shin KH, Kim J, Yoon SH, Cho JY, et al. Assessment of the drug-drug interactions between fimasartan and hydrochlorothiazide in healthy volunteers. J Cardiovasc Pharmacol. 2012;59(1):84-91.

21. Chi YH, Lee H, Paik SH, Lee JH, Yoo BW, Kim JH, et al. Safety, tolerability, pharmacokinetics, and pharmacodynamics of fimasartan following single and repeated oral administration in the fasted and fed states in healthy subjects. Am J Cardiovasc Drugs Drugs Devices Other Interv. 2011;11(5):335-46.

22. Lee HW, Lim MS, Seong SJ, Lee J, Park J, Seo JJ, et al. Effect of age on the pharmacokinetics of fimasartan (BR-A-657). Expert Opin Drug Metab Toxicol. 2011;7(11):1337-44.

23. Zou P, Yu Y, Zheng N, Yang Y, Paholak HJ, Yu LX, et al. Applications of human pharmacokinetic prediction in first-inhuman dose estimation. AAPS J. 2012;14(2):262-81.

24. Strandgarden K, Hoglund P, Gronquist L, Svensson L, Gunnarsson PO. Absorption and disposition including enterohepatic circulation of $(14 \mathrm{C})$ roquinimex after oral administration to healthy volunteers. Biopharm Drug Dispos. 2000;21(2):53-67.

25. Ouellet DM, Pollack GM. Biliary excretion and enterohepatic recirculation of morphine-3-glucuronide in rats. Drug Metab Dispos. 1995;23(4):478-84.

26. Sherwin CM, Sagcal-Gironella AC, Fukuda T, Brunner HI, Vinks AA. Development of population PK model with enterohepatic circulation for mycophenolic acid in patients with childhood-onset systemic lupus erythematosus. Br J Clin Pharmacol. 2012;73(5):727-40.

27. Wajima T, Yano Y, Oguma T. A pharmacokinetic model for analysis of drug disposition profiles undergoing enterohepatic circulation. J Pharm Pharmacol. 2002;54(7):929-34.

28. Lehr T, Staab A, Tillmann C, Trommeshauser D, Schaefer HG, Kloft C. A quantitative enterohepatic circulation model: development and evaluation with tesofensine and meloxicam. Clin Pharmacokinet. 2009;48(8):529-42.

29. Shin BS, Kim TH, Paik SH, Chi YH, Lee JH, Tan HK, et al. Simultaneous determination of fimasartan, a novel antihypertensive agent, and its active metabolite in rat plasma by liquid chromatography-tandem mass spectrometry. Biomed Chromatogr. 2011;25(11):1208-14.

30. Bulitta JB, Bingolbali A, Shin BS, Landersdorfer CB. Development of a new pre- and post-processing tool (SADAPT-TRAN) for nonlinear mixed-effects modeling in S-ADAPT. AAPS J. 2011;13(2):201-11.

31. Bulitta JB, Duffull SB, Kinzig-Schippers M, Holzgrabe U, Stephan U, Drusano GL, et al. Systematic comparison of the population pharmacokinetics and pharmacodynamics of piperacillin in cystic fibrosis patients and healthy volunteers. Antimicrob Agents Chemother. 2007;51(7):2497-507.

32. Bauer RJ, Guzy S, Ng C. A survey of population analysis methods and software for complex pharmacokinetic and pharmacodynamic models with examples. AAPS J. 2007;9(1):E60-83.

33. Bulitta JB, Landersdorfer CB. Performance and robustness of the Monte Carlo importance sampling algorithm using 
parallelized S-ADAPT for basic and complex mechanistic models. AAPS J. 2011;13(2):212-26.

34. Brendel K, Comets E, Laffont C, Laveille C, Mentre F. Metrics for external model evaluation with an application to the population pharmacokinetics of gliclazide. Pharm Res. 2006;23(9):2036-49.

35. Bulitta JB, Zhao P, Arnold RD, Kessler DR, Daifuku R, Pratt J, et al. Mechanistic population pharmacokinetics of total and unbound paclitaxel for a new nanodroplet formulation versus Taxol in cancer patients. Cancer Chemother Pharmacol. 2009;63(6):1049-63.

36. Sheiner LB, Beal SL. Some suggestions for measuring predictive performance. J Pharmacokinet Biopharm. 1981;9(4):503-12.

37. Lee J, Han S, Jeon S, Hong T, Yim DS. Pharmacokineticpharmacodynamic model of fimasartan applied to predict the influence of a high fat diet on its blood pressure-lowering effect in healthy subjects. Eur J Clin Pharmacol. 2013;69(1):11-20.

38. Ghibellini G, Vasist LS, Leslie EM, Heizer WD, Kowalsky RJ, Calvo BF, et al. In vitro-in vivo correlation of hepatobiliary drug clearance in humans. Clin Pharmacol Ther. 2007;81(3):406-13.

39. Davies B, Morris T. Physiological parameters in laboratory animals and humans. Pharm Res. 1993;10(7):1093-5.

40. Younis IR, Malone S, Friedman HS, Schaaf LJ, Petros WP. Enterohepatic recirculation model of irinotecan (CPT-11) and metabolite pharmacokinetics in patients with glioma. Cancer Chemother Pharmacol. 2009;63(3):517-24.

41. Cremers S, Schoemaker R, Scholten E, den Hartigh J, KonigQuartel J, van Kan E, et al. Characterizing the role of enterohepatic recycling in the interactions between mycophenolate mofetil and calcineurin inhibitors in renal transplant patients by pharmacokinetic modelling. Br J Clin Pharmacol. 2005;60(3):249-56.

42. Li R, Ghosh A, Maurer TS, Kimoto E, Barton HA. Physiologically based pharmacokinetic prediction of telmisartan in human. Drug Metab Dispos. 2014;42(10):1646-55.

43. Steimer JL, Plusquellec Y, Guillaume A, Boisvieux JF. A timelag model for pharmacokinetics of drugs subject to enterohepatic circulation. J Pharm Sci. 1982;71(3):297-302.

44. Colburn WA, Hirom PC, Parker RJ, Milburn P. A pharmacokinetic model for enterohepatic recirculation in the rat: phenolphthalein, a model drug. Drug Metab Dispos. 1979;7(2):100-2.

45. Jiao Z, Ding JJ, Shen J, Liang HQ, Zhong LJ, Wang Y, et al. Population pharmacokinetic modelling for enterohepatic circulation of mycophenolic acid in healthy Chinese and the influence of polymorphisms in UGT1A9. Br J Clin Pharmacol. 2008;65(6):893-907.

46. Funaki T. Enterohepatic circulation model for population pharmacokinetic analysis. J Pharm Pharmacol. 1999;51(10):1143-8.

47. Moon YJ, Sagawa K, Frederick K, Zhang S, Morris ME. Pharmacokinetics and bioavailability of the isoflavone biochanin A in rats. AAPS J. 2006;8(3):E433-42.

48. Sherwin CM, Fukuda T, Brunner HI, Goebel J, Vinks AA. The evolution of population pharmacokinetic models to describe the enterohepatic recycling of mycophenolic acid in solid organ transplantation and autoimmune disease. Clin Pharmacokinet. 2011;50(1):1-24.

49. Berg AK, Mandrekar SJ, Ziegler KL, Carlson EC, Szabo E, Ames MM, et al. Population pharmacokinetic model for cancer chemoprevention with sulindac in healthy subjects. J Clin Pharmacol. 2013;53(4):403-12.

50. de Winter BC, van Gelder T, Sombogaard F, Shaw LM, van Hest RM, Mathot RA. Pharmacokinetic role of protein binding of mycophenolic acid and its glucuronide metabolite in renal transplant recipients. J Pharmacokinet Pharmacodyn. 2009;36(6):541-64.

51. Rosner GL, Panetta JC, Innocenti F, Ratain MJ. Pharmacogenetic pathway analysis of irinotecan. Clin Pharmacol Ther. 2008;84(3):393-402.
52. Shou M, Lu W, Kari PH, Xiang C, Liang Y, Lu P, et al. Population pharmacokinetic modeling for enterohepatic recirculation in Rhesus monkey. Eur J Pharm Sci. 2005;26(2):15161.

53. Plusquellec Y, Houin G. Drug recirculation model with multiple cycles occurring at unequal time intervals. J Biomed Eng. 1992;14(6):521-6.

54. Plusquellec Y, Barre J, de Biasi J, Trenque T, Tillement JP, Houin G. Application of a pharmacokinetic model with multiple enterohepatic cycles to a new inotropic drug after infusion and oral administration. J Pharm Sci. 1992;81(10):1020-3.

55. Ibarra M, Vazquez M, Fagiolino P. Population pharmacokinetic model to analyze nevirapine multiple-peaks profile after a single oral dose. J Pharmacokinet Pharmacodyn. 2014;41(4):363-73.

56. Bastian G, Barrail A, Urien S. Population pharmacokinetics of oxaliplatin in patients with metastatic cancer. Anticancer Drugs. 2003;14(10):817-24.

57. Colom H, Lloberas N, Andreu F, Caldes A, Torras J, Oppenheimer F, et al. Pharmacokinetic modeling of enterohepatic circulation of mycophenolic acid in renal transplant recipients. Kidney Int. 2014;85(6):1434-43.

58. Ezzet F, Krishna G, Wexler DB, Statkevich P, Kosoglou T, Batra VK. A population pharmacokinetic model that describes multiple peaks due to enterohepatic recirculation of ezetimibe. Clin Ther. 2001;23(6):871-85.

59. Moon YJ, Wang L, DiCenzo R, Morris ME. Quercetin pharmacokinetics in humans. Biopharm Drug Dispos. 2008;29(4):205-17.

60. Huntjens DR, Strougo A, Chain A, Metcalf A, Summerfield S, Spalding DJ, et al. Population pharmacokinetic modelling of the enterohepatic recirculation of diclofenac and rofecoxib in rats. Br J Pharmacol. 2008;153(5):1072-84.

61. Shin BS, Hwang SW, Bulitta JB, Lee JB, Yang SD, Park JS, et al. Assessment of bisphenol A exposure in Korean pregnant women by physiologically based pharmacokinetic modeling. J Toxicol Environ Health A. 2010;73(21-22):1586-98.

62. Ploeger B, Mensinga T, Sips A, Meulenbelt J, DeJongh J. A human physiologically-based model for glycyrrhzic acid, a compound subject to presystemic metabolism and enterohepatic cycling. Pharm Res. 2000;17(12):1516-25.

63. Moriwaki T, Yasui H, Yamamoto A. A recirculatory model with enterohepatic circulation by measuring portal and systemic blood concentration difference. J Pharmacokinet Pharmacodyn. 2003:30(2):119-44.

64. Back DJ, Rogers SM. Review: first-pass metabolism by the gastrointestinal mucosa. Aliment Pharmacol Ther. 1987;1(5):339-57.

65. Ilett KF, Tee LB, Reeves PT, Minchin RF. Metabolism of drugs and other xenobiotics in the gut lumen and wall. Pharmacol Ther. 1990;46(1):67-93.

66. Ohno S, Nakajin S. Determination of mRNA expression of human UDP-glucuronosyltransferases and application for localization in various human tissues by real-time reverse transcriptase-polymerase chain reaction. Drug Metab Dispos. 2009;37(1):32-40.

67. Iwamoto K, Klaassen CD. First-pass effect of morphine in rats. J Pharmacol Exp Ther. 1977;200(1):236-44.

68. Koster AS, Frankhuijzen-Sierevogel AC, Noordhoek J. Distribution of glucuronidation capacity (1-naphthol and morphine) along the rat intestine. Biochem Pharmacol. 1985;34(19):352732.

69. Horkovics-Kovats S, Zlatos P. Asymptotics and bioavailability in multicompartment pharmacokinetic models with enterohepatic circulation. Math Biosci. 2003;184(1):69-99.

70. Horkovics-Kovats S, Zlatos P. Asymptotics and bioavailability in a 17-compartment pharmacokinetic model with enterohepatic circulation and remetabolization. Math Biosci. 2006;203(1):19-36. 